\title{
An inter-laboratory comparison to evaluate the suitability of EN 1787 standard to detect irradiation in plant-origin foods with health benefits
} \author{
M. Tomaiuolo ${ }^{c}$, C. Boniglia ${ }^{\mathrm{a}}$ \\ ${ }^{a}$ Istituto Superiore di Sanità, Roma, Italy \\ ${ }^{\mathrm{b}}$ Istituto Zooprofilattico Sperimentale della Sicilia, Palermo, Italy \\ ${ }^{\mathrm{c}}$ Istituto Zooprofilattico Sperimentale della Puglia e della Basilicata, Foggia, Italy \\ ${ }^{\mathrm{d}}$ Istituto Zooprofilattico Sperimentale della Sardegna, Sassari, Italy \\ e Istituto Zooprofilattico Sperimentale del Lazio e della Toscana, Roma, Italy \\ ${ }^{\mathrm{f}}$ Università degli Studi di Palermo, Italy
}

E. Bortolin ${ }^{\mathrm{a}, *}$, C. Cardamone ${ }^{\mathrm{b}}$, A.E. Chiaravalle ${ }^{\mathrm{c}}$, B. Carratù ${ }^{\mathrm{a}}$, G. Deiana ${ }^{\mathrm{d}}$, A. Di Noto ${ }^{\mathrm{b}}$, M.T. Di Schiavi ${ }^{\mathrm{e}}$, M.C. D'Oca ${ }^{\mathrm{f}}$, R. Gargiulo ${ }^{\mathrm{a}}$, M. Mangiacotti ${ }^{\mathrm{c}}$, G. Marchesani ${ }^{\mathrm{c}}$, M.C. Quattrini ${ }^{\mathrm{a}}$,

\section{A R T I C L E I N F O}

\section{Keywords:}

Irradiated food

Plant food supplements

PFS

Electron Spin Resonance

ESR

Nuts

Fresh blueberries

\begin{abstract}
A B S T R A C T
This paper reports the results of a study carried out to verify the applicability of the EN 1787 method, which uses the Electron Spin Resonance (ESR) technique for the identification of irradiated plant-origin foods with health benefits. The method was tested on samples of herbal ingredients of Plant Food Supplements (PFSs), nuts and fresh blueberries. Untreated and irradiated samples of Camellia sinensis (leaves) Ginkgo biloba (leaves), Glycine max (seeds), Silybum marianum (fruits), Vaccinium myrtillus (fruits), almonds, hazelnuts, peanuts, pistachios, walnuts and fresh blueberries were analysed. The work includes an inter-laboratory blind test involving five Italian laboratories that perform routine analyses for the official control of irradiated food. A total of 180 untreated and irradiated samples of PFS ingredients, nuts and fresh blueberries were analysed. The analyses on the irradiated samples were replicated even a long time after irradiation (up to two years depending on the matrix) to test the reliability of the method throughout the shelf life of the products.

The results were matrix-dependent: all the $5 \mathrm{kGy}$ irradiated nuts and the $1 \mathrm{kGy}$-irradiated blueberries were correctly classified, whereas herbal ingredients showed complex ESR spectra with spurious signals which often prevented the correct classification of the sample.
\end{abstract}

\section{Introduction}

Plants growing in an open environment are exposed to chemical and microbiological hazards. Since there is not a cleansing step that can remove all contaminants, the prevention of contamination during the production stages is of outmost importance (Piniero \& Diaz, 2007). To preserve the hygienic quality and ensure shelf life, the treatment of foodstuffs with ionising radiation has been largely used against microbial deterioration during transport and storage. (FAO/WHO, 2002; FAO/WHO, 2004; Kume, Furuta, Todoriki, Uenoyama, \& Kobayashi, 2009; Kume \& Todoriki, 2013; Ihsanullah \& Rashid, 2017; Roberts, 2014; WHO, 1999). In the European Union food irradiation is regulated by Directive, 1999 and Directive, 1999, which state that the treatment foods are subject to must be indicated. Every year, all Member States are to notify the European Commission of the results of the tests performed to detect irradiated foods, and check the related labelling. EN 1787,2000 is one of the analytical methods set out by the European Committee for Standardisation for the official control of irradiated foods. It uses the Electron Spin Resonance (ESR) technique to identify the radical induced in cellulose by ionising radiation; this radical yields an ESR spectrum characterised by two weak satellite lines at a specific distance (about $6.0 \mathrm{mT}$ ) from each other and symmetrically located on the sides of an intense peak detectable in untreated samples as well. The central peak, whose intensity strongly increases after irradiation, is indeed the results of the superposition of different signals including the central component of the two satellite lines (Bortolin, Bustos Griffin, Cruz-Zaragoza, De Coste, \& Onori, 2006; Chiappinelli et al., 2019; Franco et al., 2004; Tomaiuolo et al., 2018). The application of the

\footnotetext{
* Corresponding author. Core Facilities, Istituto Superiore di Sanità, Rome, Italy.

E-mail address: emanuela.bortolin@iss.it (E. Bortolin).
} 
method is simple and time-saving: no laborious sample preparation is required and the measurement usually takes a few minutes. In addition, once validated the method does not require confirmation of the nonnegative results. The method has been internationally validated through trials for pistachio nut shells, strawberries and paprika (Linke et al., 1995; Linke et al., 1996; Raffi, 1992; Raffi, Stevenson, Kent, Thiery, \& Belliardo, 1992; Schreiber et al., 1993; Schreiber et al., 1996) but, in principle, it could be applicable to all vegetables containing cellulose (Chiappinelli et al., 2019; Jeong, Akram, Ahn, \& Kwon, 2014; Jo, Kyung, Park, \& Kwon, 2016; Maghraby, Salama, Sami, Mansour, \& El Sayed, 2014; Raffi et al., 1989; Sezer, Ece, Uslu, Osmen, \& Savin, 2019; Shahbaz, Akram, Ahn, \& Kwon, 2013; Tomaiuolo et al., 2018). In practice, however, its reliability in detecting irradiation is limited when the sample has a low content of crystalline cellulose and/or a high moisture content, which favours radical recombination leading to false negative results (Bortolin, Bustos Griffin, Cruz-Zaragoza, De Coste, \& Onori, 2006; Kwon, Ahn, Akram, Son, \& Lee, 2013; Yordanov, Lagunov, \& Dimov, 2009). Moisture is a limitation especially in water-containing matrices, e.g., fresh fruit, for the fast fading of the signal yields false negatives. Recently, however, false positive classifications have been made for nuts (hazelnuts and walnuts): owing to shell bleaching by chemical reagents, ESR signals look similar to those induced by ionising radiation (Butz \& Hildebrand, 2006; Werner, Straub, Zumsteeg, \& Kuntz, 2020). Problems were also met when applying the method to herbs and spices because the presence of intrinsic radicals responsible for "spurious" signals makes the ESR spectra complex and difficult to interpret (Ahn, Akram, Jo, \& Kwon, 2012; Kim, Ahn, Shahbaz, Kim, \& Kwon, 2014; Sanyal et al., 2014; Yordanov et al., 2009).

Given such limitations, for a correct application of the method in official controls it is critical to investigate the characteristics of the ESR spectra of each single matrix or group of matrices, as well as monitor their signal stability during the shelf life of the product. The present work aimed to test the applicability of EN 1787 Standard for the identification of plant-origin food with health benefits, such as fruit and herbal ingredients for PFSs which are largely consumed all around the word. To this purpose, samples were analysed of nuts (almonds, hazelnuts, peanuts, pistachios and walnuts), fresh blueberries and herbs, namely Camellia sinensis, Ginkgo biloba, Glycine max, Silybum marianum, Vaccinium myrtillus, in the forms of dried raw material (leaves, fruits, seeds) and dried extract. Reliable analytical methods are urgently needed since official checks in Europe on products at the marketing stage have found that non-negligible percentages of plant food supplements (PFSs) and their ingredients were irradiated and incorrectly labeled. (http://ec.europa.eu/food/safety/biosafety/irradiation/ reports; Boniglia, Aureli, Bortolin, \& Onori, 2009).

The present work involved five Italian laboratories engaged in the official control of irradiated foods. After a preliminary intra-laboratory validation of the analytical procedure applied to the matrices chosen for the study, an inter-laboratory blind test was organised, where 180 untreated and irradiated samples of PFS ingredients, nuts and fresh blueberries were analysed. The irradiated samples were analysed even a long time (up to two years depending on the matrix) after irradiation to test the applicability of the method throughout the shelf life of the products. The irradiation dose values for the different samples were chosen among those commonly used all over the world. The applied doses depend on the food items and on the purpose of irradiation; in particular, herbal ingredients and nuts are irradiated at doses ranging from 3 to $10 \mathrm{kGy}$ to eliminate microbial pathogens, insect pests, mold, and other spoilage organisms, whereas fresh blueberries at doses ranging from 0.15 to $1 \mathrm{kGy}$ for phytosanitary purposes against fruit flies and other critical plant pests. For this study dried herbal ingredients and nuts were irradiated at $5 \mathrm{kGy}$, and blueberries at 0.15 and $1 \mathrm{kGy}$. In the preliminary intra-laboratory step of the work herbal ingredients were irradiated also at $1 \mathrm{kGy}$ to test the sensitivity of the method in detecting lower doses than those normally applied to these matrices.

\section{Materials and methods}

\subsection{Samples}

The matrices were PFS ingredients, nuts and fresh fruits. All the products were not labeled as treated by ionizing radiation, nevertheless analyses were performed by EN 1788 method on all the samples as received to check if they had been irradiated and not correctly labeled. Before analysis all the products were stored at room conditions inside their original packaging.

\subsubsection{PFS ingredients}

Five dried herbal ingredients were chosen from among the most commonly used for PFSs, in the form of both raw materials (leaves, fruits, seeds) and herbal extracts: Camellia sinensis (leaves), Ginkgo biloba (leaves), Glycine max (seeds), Silybum marianum (fruits), Vaccinium myrtillus (fruits) were purchased from herbalists whereas the corresponding herbal extracts were produced by Indena S.p.A (Italy). For each product, the analyses were performed on untreated and irradiated samples.

\subsubsection{Fruit}

Samples of almonds, hazelnuts, peanuts, pistachios, walnuts and fresh blueberries were purchased at the local markets in different Italian regions.

\subsection{Irradiation}

Sample irradiation was performed at room temperature with a lowenergy X-ray irradiator (RS $2400 \mathrm{Rad}$ source Inc.) operating at $150 \mathrm{kV}$ and $45 \mathrm{~mA}$ with a dose rate of $40 \mathrm{~Gy} \mathrm{~min}^{-1}$ as measured with a calibrated ion chamber (Rad-cal Inc.). The technical characteristics of this facility (radiation quality and energy), compatible with those required by the European legislation, allowed to simulate a real radiation treatment in an industrial plant. The uniformity of the dose was obtained by irradiating the food matrices in sample holders inside a carousel rotating around the X-ray tube. Dosimetric properties were tested by the Electron Spin Resonance alanine pellet system. The absorbed doses hereby reported are doses to water measured by alanine dosimeters with a diameter of $5 \mathrm{~mm}$ (Bruker, Rheinstetten, Germany) and an uncertainty of about $5 \%$. The products were irradiated and stored, at room conditions of temperature and humidity, in light-protected containers. Dried herbal ingredients were irradiated at 1 and $5 \mathrm{kGy}$, nuts at $5 \mathrm{kGy}$ and blueberries at 0.1 and $1 \mathrm{kGy}$ (Table 1 ).

Table 1

Scheme of the intra-laboratory validation test.

\begin{tabular}{|c|c|c|c|c|}
\hline Category & Matrix & $\begin{array}{l}\text { Dose } \\
\text { (kGy) }\end{array}$ & $\begin{array}{l}\text { Monitoring } \\
\text { period }\end{array}$ & $\begin{array}{l}\text { No. of } \\
\text { laboratories }\end{array}$ \\
\hline PFSs & $\begin{array}{l}\text { Camellia sinensis } \\
\text { Ginkgo biloba } \\
\text { Glycine max } \\
\text { Silybum } \\
\text { marianum } \\
\text { Vaccinium } \\
\text { myrtillus }\end{array}$ & $1 ; 5$ & 2 years & 3 \\
\hline Nuts & $\begin{array}{l}\text { Almonds } \\
\text { Hazelnuts } \\
\text { Peanuts } \\
\text { Pistachios } \\
\text { Walnuts }\end{array}$ & 5 & 2 years & 4 \\
\hline Fresh fruit & Blueberries & $0.15 ; 1$ & 3 weeks & 4 \\
\hline
\end{tabular}




\subsection{ESR analysis}

\subsubsection{Intra-laboratory validation tests}

For the analyses, four laboratories followed the procedure described in the EN 1787 standard, according to the scheme reported in Table 1. All four laboratories carried out tests on nuts and fresh blueberries on untreated samples and on samples irradiated with doses of $0.15 \mathrm{kGy}$ and $1 \mathrm{kGy}$ (blueberries), and $5 \mathrm{kGy}$ (nuts). Three of them also analysed PSF ingredients (raw materials and extracts) in untreated samples and samples irradiated at $1 \mathrm{kGy}$ and $5 \mathrm{kGy}$.

The validation tests included checks of signal stability under normal storage conditions in order to verify the reliability of the method during the shelf life of the products. The measurements were thus periodically repeated on the same aliquot since the ESR measurement does not cause the disappearance of the signal. After irradiation, signal stability was checked once or two/three times a week, depending on the rate of decrease. ESR signals of PFS ingredients and nuts were monitored up to two years after irradiation, while on blueberries the measurements were repeated more often but always within three weeks (more or less their shelf life). The results obtained by the participants were compared, and an analytical procedure was agreed upon.

\subsubsection{Inter-laboratory validation test}

To validate the ESR procedure elaborated during the intra-laboratory validation step, a blind test was carried out, involving five laboratories (Table 2). A total number of 180 samples (75 PFS ingredients, 75 nuts and 30 fresh blueberry samples) were prepared and identified with a specific code. Specifically, 25 untreated and 50 irradiated (5 kGy) samples of PFS ingredients, 25 untreated and 50 irradiated ( $5 \mathrm{kGy}$ ) nut samples, and 10 untreated, 10 irradiated at $0.15 \mathrm{kGy}$ and 10 irradiated at $1 \mathrm{kGy}$ blueberry samples were prepared and sent to the laboratories. Irradiation and shipping were completed within a week. The majority of the samples were irradiated just before shipping. To confirm the stability of the signal and the reliability of the method throughout shelf life, 25 out of the 50 irradiated PFS ingredients and 25 out of the 50 irradiated nuts samples were taken from among those treated with ionizing radiation about two years before for the intralaboratory tests. Regarding PFS ingredients, as the ESR method turned out to be unsuitable for detecting herbal extracts during the preliminary tests, the trial was limited to raw materials.

\subsubsection{Sample preparation}

For ESR measurement, about $100 \mathrm{mg}$ of PFS ingredients and nut shells were cut, where necessary, into pieces small enough for the ESR tube. Measurements for Glycine max were performed on both whole seeds and outer peel. Fresh blueberries were prepared following the

Table 2

Scheme of the inter-laboratory blind test.

\begin{tabular}{lllll}
\hline Category & Matrix & $\begin{array}{l}\text { Dose } \\
(\mathrm{kGy})\end{array}$ & $\begin{array}{l}\text { No. of } \\
\text { samples }\end{array}$ & No. of laboratories \\
\hline PFSs & $\begin{array}{l}\text { Camellia sinensis } \\
\text { Ginkgo biloba } \\
\text { Glycine max } \\
\text { Silybum marianum } \\
\text { Vaccinium } \\
\text { myrtillus } \\
\text { Almonds }\end{array}$ & 5 & $75^{\mathrm{a}}$ & 5 \\
Nuts & 5 & $75^{\mathrm{a}}$ & 5 \\
Hazelnuts & $\begin{array}{l} \\
\text { Peanuts } \\
\text { Pistachios } \\
\text { Walnuts } \\
\text { Blueberries }\end{array}$ & $0.15 ; 1$ & 30 & 5 \\
\hline
\end{tabular}

a 25 out of 50 irradiated PFS ingredients and 25 out of 50 irradiated nut samples had been treated with ionizing radiation about two years before the analysis. procedure described for strawberries in EN 1787. Before measurement, the samples were kept at about $45{ }^{\circ} \mathrm{C}$ for about $1 \mathrm{~h}$ to reduce the moisture content of the matrices.

\subsubsection{ESR measurements}

ESR measurements were performed at room temperature and humidity with different models of Bruker spectrometers operating in the $\mathrm{X}$ band: Elexsys with a Bruker Super High Q cavity (one laboratory), EScan Food Analyzer (three laboratories) and EMX with a Bruker Very High Quality cavity (one laboratory). Parameters were set as indicated in the EN 1787 standard. Frequency: 9.78-9.93 GHz, depending on the equipment; center field: about $350 \mathrm{mT}$; sweep width: about $20 \mathrm{mT}$; microwave power: $0.4-0.8 \mathrm{~mW}$; modulation amplitude: $0.4-0.9 \mathrm{mT}$; sweep speed: $5 \mathrm{mT} / \mathrm{min}-10 \mathrm{mT} / \mathrm{min}$; number of scans: $3-64$, depending on the equipment. The samples were inserted in Suprasil tubes with a diameter of $4-5 \mathrm{~mm}$.

\section{Results and discussion}

\subsection{Intra-laboratory validation tests}

These tests showed that ESR spectra of plant-origin foods are generally complex and difficult to interpret, owing to the presence of different radical species in the chemical composition of these matrices. The classification of the samples was unambiguous for nuts and $1 \mathrm{kGy}-$ irradiated blueberries, whereas PFS ingredient spectra showed overlapping signals which made it difficult to classify the samples. On the whole, the results obtained by the participating laboratories were in agreement, and a common analytical procedure was set up to be used for the inter-laboratory blind test.

\subsubsection{PFS ingredients}

Fig. 1 shows the ESR spectra of the untreated products. As expected, in agreement with the EN 1787 standard, in all the untreated samples of raw materials a symmetric signal was always well visible, with intensity depending on the specific matrix. Besides that, in most of the samples other unidentifiable peaks, but certainly not attributable to irradiated cellulose, were visible on each side of the central signal.

Difficult to interpret, complex spectra were recorded with samples irradiated at $1 \mathrm{kGy}$. Better results were obtained with samples re-irradiated at $5 \mathrm{kGy}$ whose spectra were easier to interpret. Irradiated Glycine max gave the best results (Fig. 2), especially when only the outer peel of the seeds was used. The satellite lines, specific of irradiation, were visible also in the irradiated samples of Silybum marianum but often the left line appeared not completely resolved, as shown in Fig. 3. Conversely, Camellis sinensis (Fig. 4) Ginkgo biloba (Fig. 5) and Vaccinium myrtillus (Fig. 6) showed spectra where such lines were not always detectable.

The spectra showed no significant shape variations with time, and the satellite lines, if any, were well visible more or less throughout the entire observation period, depending on the matrix and the characteristics (sensitivity and calibration) of the measurement equipment. Herbal extracts yielded unsatisfactory results: the spectra were extremely complex, with multiple peaks, and the radiation-induced signal could not be identified in any of the samples, regardless of the dose.

\subsubsection{Fruit}

The samples of almonds, hazelnuts, pistachios, walnuts, irradiated (5 kGy) and untreated, were all properly identified; their spectra appeared very similar to those reported in the EN standard 1787, with the satellite lines well visible in the case of irradiated samples. No false positive results were obtained with hazelnuts and walnuts, which indicated that the nuts had not been bleached. As expected and recently reported for hazelnuts and walnuts by Chiappinelli et al. (2019) and Tomaiuolo et al. (2018), the intensity of the satellite lines decreased with time (Fig. 7), but they were still visible for a long time (two years 

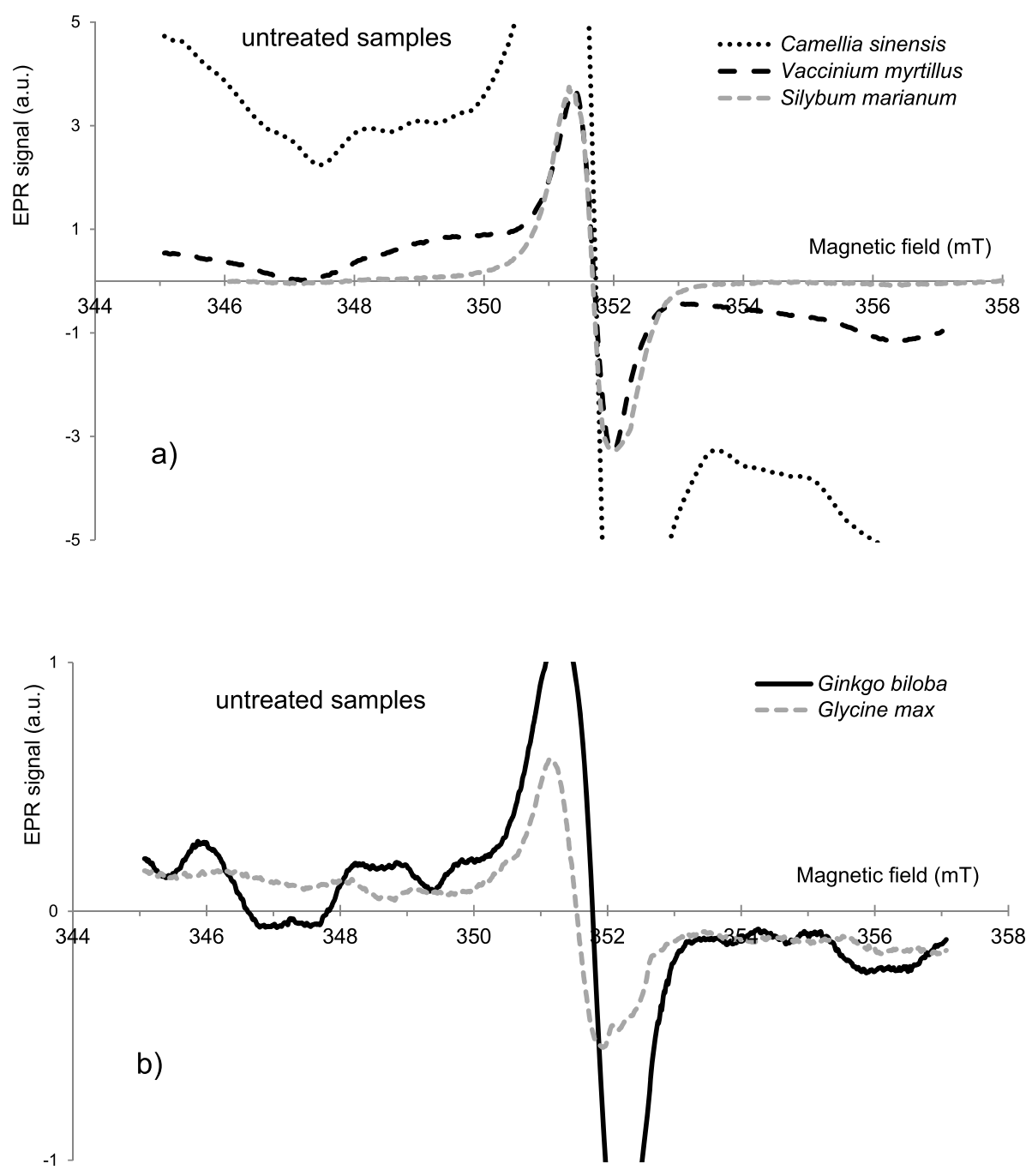

Fig. 1. ESR spectra of untreated samples.

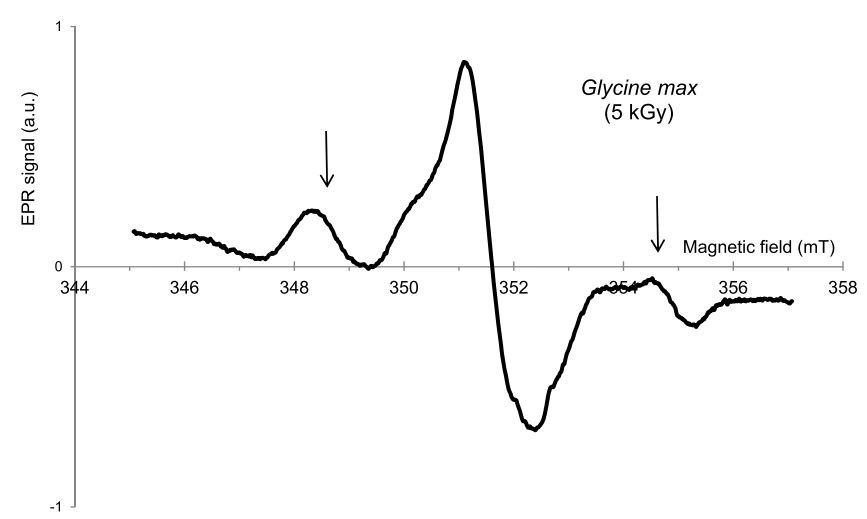

Fig. 2. ESR spectra of a sample of Glycine max (seeds) after irradiation at $5 \mathrm{kGy}$. The satellite lines are well evident.

after irradiation and more). As an example, Fig. 8 reports the ESRspectra of irradiated almonds recorded at different times after irradiation. Fig. 8. b shows an evident decrease also of the central peak which supports the presence in that structure of not stable radiation-induced signals already reported in literature and, in particular, of a central line belonging to the same structure of the satellite lines, as confirmed by Chiappinelli et al. (2019) and Tomaiuolo et al. (2018).

Peanuts provided slightly different results; 2 out of 15 samples were

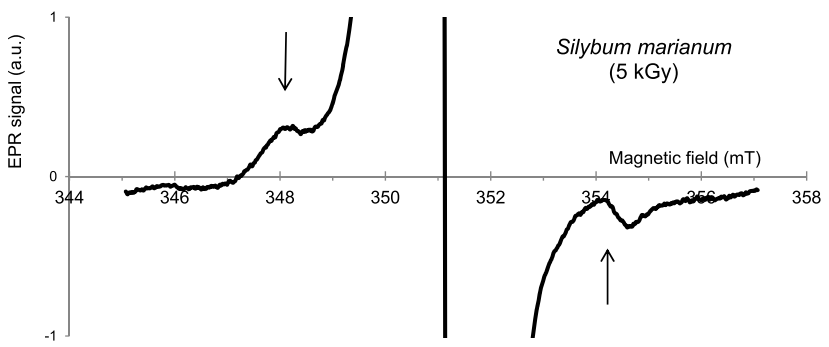

Fig. 3. ESR spectra of a sample of Silybum marianum (fruits) after irradiation at $5 \mathrm{kGy}$. The satellite lines are visible but the left one is not completely resolved.

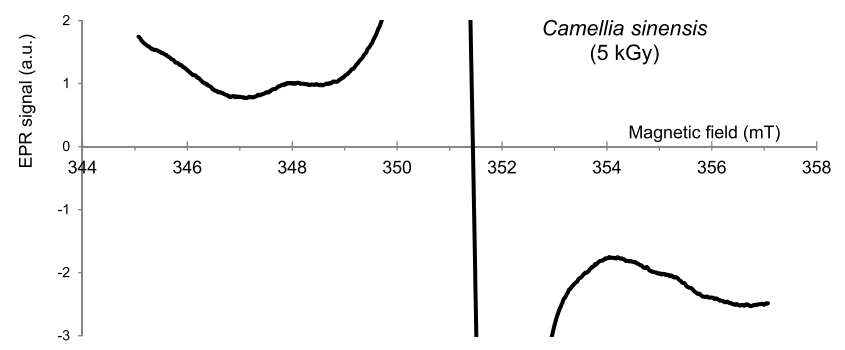

Fig. 4. ESR spectra of a sample of Camellia sinensis (leaves) after irradiation at $5 \mathrm{kGy}$. The satellite lines are not cleraly visible. 


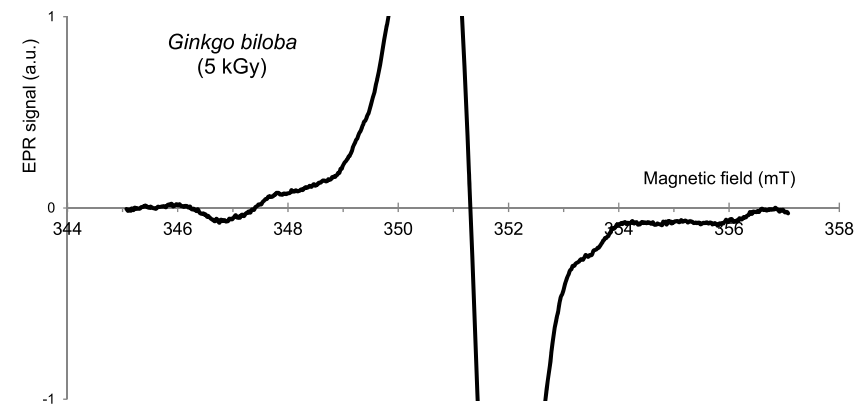

Fig. 5. ESR spectra of a sample of Ginkgo biloba (leaves) after irradiation at $5 \mathrm{kGy}$. The satellite lines cannot be detected.

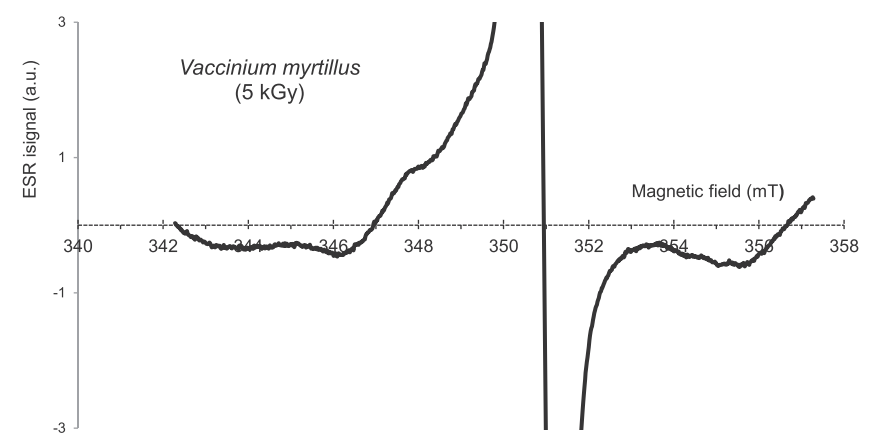

Fig. 6. ESR spectra of a sample of Vaccinium myrtillus (fruits) after irradiation at 5 kGy. The satellite lines cannot be detected.

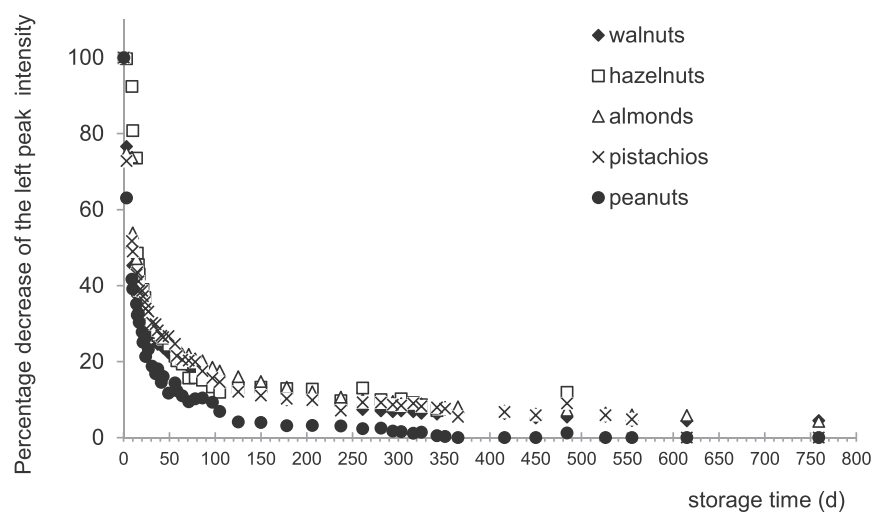

Fig. 7. Percentage decrease of the left satellite line of $5 \mathrm{kGy}$ irradiated nuts. Each value is the mean of three measurements repeated on the same aliquot; the error bar is smaller than the symbol used in graph.

not correctly identified and the fading was faster (Fig. 7). Moreover, the characteristics of the spectrum were also slightly different as shown in Fig. 9, where the distance between satellite lines is reported for the various nuts. The values in the graph were obtained from measurements repeated on the same aliquot at different times after irradiation. The value for such distance is clearly higher for peanuts than for the other nuts. This is likely attributable to the specific composition of peanut shells. As a point of fact, peanuts are classified as legumes, not nuts.

Fresh blueberries were analysed at different times within 3 weeks, which corresponds more or less to their shelf life. The samples irradiated at $1 \mathrm{kGy}$ were correctly identified, whereas the ones irradiated at a low dose $(0.15 \mathrm{kGy})$ all showed similar spectra in which only the satellite line on the right could be seen (Fig. 10).
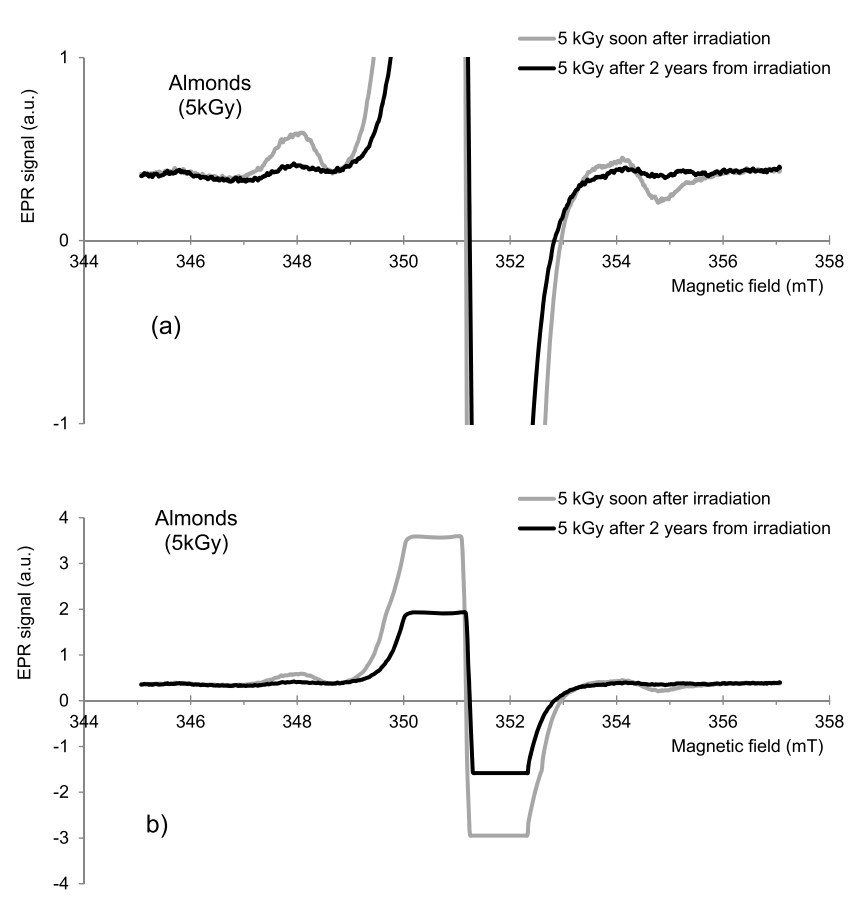

Fig. 8. ESR spectra of a sample of almonds irradiated at $5 \mathrm{kGy}$. a) The radiationinduced satellite lines are still well visible about 2 years after irradiation. b) The central peak appears strongly reduced after two years from irradiation.

\subsection{Inter-laboratory validation test}

All the matrices and the results of the ESR inter-laboratory validation test are reported in Tables 3-5.

PFS ingredients. Since the ESR method turned out to be unsuitable to detect herbal extracts during the preliminary intra-laboratory tests, the trial was limited to raw materials.

Glycine max. On the basis of the results of the preliminary tests, only the outer peel of the fruit was used.

Herbal ingredients. $80 \%$ of samples (60 out of 75 ) were correctly identified; the remaining $20 \%$ (15 samples) gave false negative (11 samples) or false positive (4 samples) classifications.

Nuts. Only 2 peanut samples (one untreated and one $5 \mathrm{kGy}$ irradiated) out of a total of 75 samples (less than $3 \%$ ) could not be correctly identified.

Fresh fruit. Blueberries were correctly identified only at $1 \mathrm{kGy}$.

These results confirmed that the ESR method can be used for the routine official checks on samples of almonds, hazelnuts, pistachios and walnuts, but not for peanuts or PFS ingredients such as Camellia sinensis, Ginkgo biloba, Glycine max, Sylibum fructus and Vaccinium myrtillus, which gave incorrect classifications. Irradiated fresh blueberries detection seemed to depend on the dose of treatment, which limits the applicability of the method to this matrix.

\section{Conclusions}

This study confirmed that the ESR spectra of plant-origin foods are generally complex and difficult to interpret as the result of a large variety of natural components, e.g., radicals. In particular, PFS ingredients showed overlapping signals due to the presence of different intrinsic radical species, which made it difficult to recognize the satellite lines typical of irradiated cellulose. The identification of the radiation-induced signal was unambiguous for nuts (with the exception of peanuts), for both untreated and irradiated samples. Fresh blueberries were correctly identified, even after 3 weeks, their shelf life approximately, but only those samples that had been irradiated at $1 \mathrm{kGy}$.

In conclusions, below some recommendation reflecting the final 


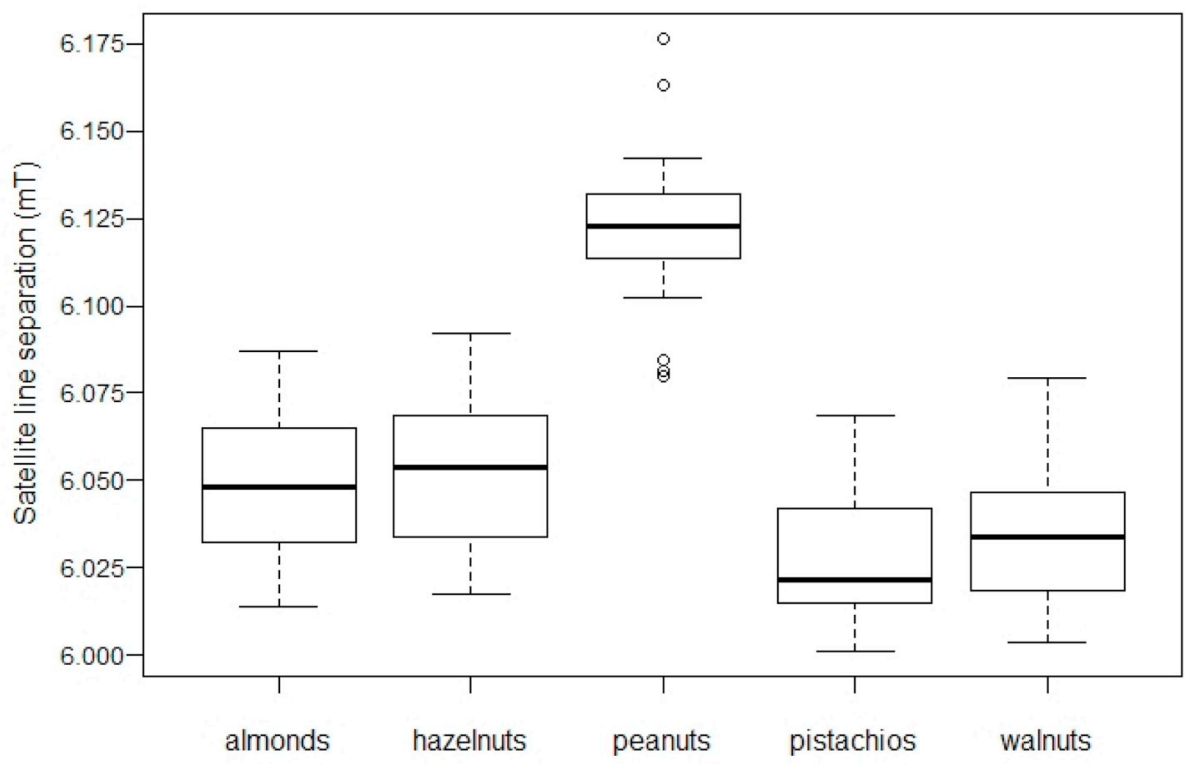

Fig. 9. Distance between satellite lines for the various nuts. The line in the box represents the median of measurements repeated on the same aliquot at different times after irradiation.

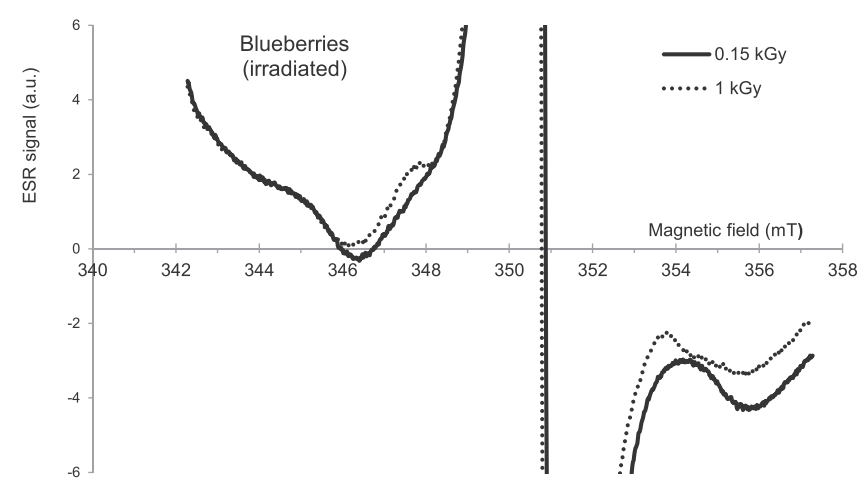

Fig. 10. ESR spectra of irradiated blueberries. The left satellite line cannot be clearly detected in the ESR spectrum of the $0.15 \mathrm{kGy}$ irradiated sample.

stage of the inter-comparison.

PFS ingredients. The results were matrix dependent. Therefore, to avoid unambiguous interpretation of the spectra, it is worth verifying the reliability of the method on each single matrix before applying it for official controls.

Nuts. The results of the inter-comparison confirmed that the ESR method can be used for routine official checks on samples of almonds, hazelnuts, pistachios and walnuts. However, further investigations should be periodically conducted to verify whether bleached samples giving false positives are present on the Italian market.
Table 4

Inter-laboratory results: nuts.

\begin{tabular}{llllll}
\hline Matrix & $\begin{array}{l}\text { untreated } \\
\text { samples }\end{array}$ & $\begin{array}{l}\text { Irradiated } \\
\text { samples }\end{array}$ & $\begin{array}{l}\text { Correctly } \\
\text { identified }\end{array}$ & $\begin{array}{l}\text { False } \\
\text { negative }^{\mathrm{a}}\end{array}$ & $\begin{array}{l}\text { False } \\
\text { positive }^{\mathrm{b}}\end{array}$ \\
\hline Almonds & 5 & 10 & 15 & 0 & 0 \\
Hazelnuts & 5 & 10 & 15 & 0 & 0 \\
Peanuts & 5 & 10 & 13 & 1 & 1 \\
Pistachios & 5 & 10 & 15 & 0 & 0 \\
Walnuts & 5 & 10 & 15 & 0 & 0 \\
Total & $\mathbf{2 5}$ & $\mathbf{5 0}$ & $\mathbf{7 3}$ & $\mathbf{1}$ & $\mathbf{1}$ \\
\hline
\end{tabular}

a false negative: irradiated samples identified as untreated

${ }^{\mathrm{b}}$ false positive: untreated samples identified as irradiated

Fresh blueberries. ESR response of these matrices resulted dose dependent. Therefore, as the treatment dose can be unknown, the method is to be considered not reliable for official control analyses on these products.

\section{Declaration of competing interest}

The corresponding author certify that all authors have NO affiliations with or involvement in any organization or entity with any financial interest (such as honoraria; educational grants; participation in speakers' bureaus; membership, employment, consultancies, stock ownership, or other equity interest; and expert testimony or patentlicensing arrangements), or non -financial interest (such as personal or

Table 3

Inter-laboratory results: PFS ingredients (raw materials).

\begin{tabular}{|c|c|c|c|c|c|}
\hline Matrix & Untreated samples & Irradiated samples & Correctly identified & False negative $^{a}$ & False positive $^{b}$ \\
\hline Camellia sinensis & 5 & 10 & 13 & 2 & 0 \\
\hline Ginkgo biloba & 5 & 10 & 9 & 5 & 1 \\
\hline Glycine $\max$ & 5 & 10 & 14 & 1 & 0 \\
\hline Silybum marianum & 5 & 10 & 13 & 0 & 2 \\
\hline Vaccinium myrtillus & 5 & 10 & 11 & 3 & 1 \\
\hline Total & 25 & 50 & 60 & 11 & 4 \\
\hline
\end{tabular}

${ }^{\text {a }}$ false negative: irradiated samples identified as untreated

b false positive: untreated samples identified as irradiated 
Table 5

Inter-laboratory results: fresh blueberries.

\begin{tabular}{|c|c|c|c|c|c|}
\hline Matrix & Untreated samples & Irradiated samples & Correctly identified samples & False negative $^{\mathrm{a}}$ & False positive $^{b}$ \\
\hline Blueberries & 10 & 20 & 22 & 8 & 0 \\
\hline Total & 10 & 20 & 22 & 8 & 0 \\
\hline
\end{tabular}

a False negative: irradiated samples identified as untreated.

b false positive: untreated samples identified as irradiated.

professional relationships, affiliations, knowledge or beliefs) in the subject matter or material discussed in this manuscript.

\section{Acknowledgments}

This research was partially funded by the Italian Ministero della Salute (Italy), grant ID: RF-2010-2314784. The authors are grateful to Ms. Monica Brocco for the English editing of the manuscript.

\section{References}

Ahn, J. J., Akram, K., Jo, D., \& Kwon, J. H. (2012). Investigation of different factors affecting the electron spin resonance-based characterization of gamma-irradiated fresh, white and red ginseng. Journal of ginseng research, 36(3), 308-312.

Boniglia, C., Aureli, P., Bortolin, E., \& Onori, S. (2009). Verification of imported food upon import for radiation processing: Dried herbs, including herbs used in food supplements, and spices by PSL and TL. Radiation Physics and Chemistry, 78, 679-681.

Bortolin, E., Bustos Griffin, E., Cruz-Zaragoza, E., De Coste, V., \& Onori, S. (2006). Electron paramagnetic resonance detection of Mexican irradiated spices. International Journal of Food Science and Technology, 41, 375-382.

Butz, B., \& Hildebrand, A. (2006). Bleichen von Walnüssen und Pistazien - einfluss auf den Bestrahlungsnachweis mittels ESR und GC/MS. DLR, Heft, 4, 154-157.

Chiappinelli, A., Mangiacotti, M., Tomaiuolo, M., Trotta, G., Marchesani, G., \& Chiaravalle, A. E. (2019). Identification of X-ray-irradiated hazelnuts by electron spin resonance (ESR) spectroscopy. European Food Research and Technology, 245(10), 2323-2329.

Directive (1999). 2/EC of the European Parliament and of the Council of 22 February 1999 on the approximation of the laws of the member states concerning foods and food ingredients treated with ionizing radiation (Directive, 1999a). Official Journal of the European Communities - Legislation, 66, 16-22.

Directive (1999). 3/EC of the European Parliament and of the Council of 22 February 1999 on the establishment of a Community list of the laws of foods and food ingredients treated with ionizing radiation (Directive, 1999b). Official Journal of the European Communities - Legislation, 66, 24-25. http://ec.europa.eu/food/safety/ biosafety/irradiation/reports_en.

EN 1787 (2000). Detection of irradiated food containing cellulose by ESR spectroscopy. Belgium. Brussels: European Committee for Standardisation.

FAO/WHO (2002). Codex alimentarius commission, joint FAO/WHO food standards pro gramme. (Budapest): Codex Committee on Methods of Analysis and Sampling.

FAO/WHO (2004). Fruit and vegetable for health. Japan: Report of a Joint FAO/WHO Workshop.

Franco, R. W. A., Martin-Neto, L., Kato, S. A., Furlan, G. R., Walder, J. M. M., \& Colnago, L. A. (2004). Identification of irradiation treatment in black pepper by electron paramagnetic resonance. International Journal of Food Science and Technology, 39, 395-401. http://ec.europa.eu/food/safety/biosafety/irradiation/reports_en.

Ihsanullah, I., \& Rashid, A. (2017). Current activities in food irradiation as a sanitary and phytosanitary treatment in the Asia and Pacific Region and a comparison with advanced countries. Food Control, 72, 345-359.

Jeong, M. S., Akram, K., Ahn, J. J., \& Kwon, J. H. (2014). Applicability of irradiation detection techniques and quality characterization of cinnamon powders available in the Korean market. International Journal of Food Properties, 17(10), 2192-2206.

Jo, Y., Kyung, H. K., Park, H. J., \& Kwon, J. H. (2016). Irradiated fruits can be identified by detecting radiation-induced markers with luminescence and ESR analyses for different trading fruits. Applied Biological Chemistry, 59, 59-65.

Kim, B. K., Ahn, J. J., Shahbaz, H. M., Kim, C. T., \& Kwon, J. H. (2014). Effect of drying treatment on physical identification characteristics of irradiated seasonings. Food Analytical Methods, 7(2), 268-275.

Kume, T., Furuta, M., Todoriki, S., Uenoyama, N., \& Kobayashi, Y. (2009). Status of food irradiation in the world. Radiation Physics and Chemistry, 78, 222-226.

Kume, T., \& Todoriki, S. (2013). Food irradiation in Asia, the European Union, and the United States: A status update. Radioisotopes, 62(5), 291-299.

Kwon, J. H., Ahn, J. J., Akram, K., Son, I. J., \& Lee, S. O. (2013). Characterization of radiation-induced luminescence properties and free radicals for the identification of different gamma-irradiated teas. Analytical and Bioanalytical Chemistry, 405, 4225-4234.

Linke, B., Helle, N., Ammon, J., Ballin, U., Brockmann, R., Brunner, J., et al. (1995). Elektronen-spinresonanz-spektroskopische Untersuchungen zur Identifizierung bestrahlter Krustentiere und Gewurze: Durchfuhrung eines Ringversuches an Nordseekrabben, Kaisergranat und Paprikapulver. Report of the Federal Institute for Health Protection of Consumer and Veterinary Medicine. BfVV-HeftBerlin: Bundesinstitut fur gesundheitlichen Verbaucherschutz und Veterinarmedizin.

Maghraby, A., Salama, E., Sami, A., Mansour, A., \& El Sayed, M. (2014). Identification and dosimetry of irradiated walnuts (Juglans regia) using EPR. Radiation Effects and Defects in Solids: Incorporating Plasma Science and Plasma Technology, 167(3), $170-178$.

Piniero, M., \& Diaz, L. B. (2007). Improving the safety and quality of fresh fruit and vegetables (ffv): A practical approach. I international symposium on fresh food quality standards: Better food by quality and assurance. ISHS Acta Horticulturae, 741.

Raffi, J., \& Agnel, J. P. (1989). Electron spin resonance identification of irradiated fruits. Radiation Physics and Chemistry, 34, 891-894.

Raffi, J., Stevenson, M. H., Kent, M., Thiery, J. M., \& Belliardo, J. J. (1992). European intercomparison on electron spin resonance identification of irradiated foodstuffs. International Journal of Food Science and Technology, 27, 111-124.

Roberts, P. B. (2014). Food irradiation is safe: Half a century of studies. Radiation Physics and Chemistry, 105, 78-82.

Sanyal, B., Ahn, J. J., Maeng, J. H., Kyung, H. K., Lim, H. K., Sharma, A., et al. (2014). An improved approach to identify irradiated spices using electronic nose, FTIR, and EPR spectroscopy. Journal of Food Science, 79(9), C1656-C1664.

Schreiber, G. A., Helle, N., Schlzki, G., Linke, B., Spielberg, A., Mager, M., et al. (1996). In C. H. McMurray, E. M. Stewart, R. Gray, \& J. Pearce (Eds.). Interlaboratory tests to identify irradiation treatment of various foodstuffs via gas chromatographic detection of hydrocarbons, ESR spectroscopy and TL analysis. Detection methods for irradiated foodsCurrent status (pp. 98-107). Cambridge, UK: Royal Society of Chemistry.

Schreiber, G A, Helle, N, Schlzki, G, Spielberg, A, Linke, B, Wagner, U, \& Bogl, K W (1993). Intercomparisons to evaluate the suitability of gaschromatografic, electron spin resonance spectrometric and thermoluminescence methods to detect irradiated foods in routine control. Radiation Physics and Chemistry, 42, 391-396.

Sezer, M. O., Ece, E., Uslu, A., Osmen, A., \& Savin, U. (2019). Determination of the irradiation effects on senna Casia acutifolia) leaves by ESR technique and microbiological analysis. Radiation Physics and Chemistry, 165108434.

Shahbaz, H. M., Akram, K., Ahn, J., \& Kwon, J. (2013). Investigation of radiation-induced free radicals and luminescence properties in fresh pomegranate fruits. Journal of Agricultural and Food Chemistry, 61, 4019-4025.

Tomaiuolo, M., Mangiacotti, M., Trotta, G., Marchesani, G., Chiappinelli, A., \& Chiaravalle, A. E. (2018). Identification of X-ray irradiated walnuts by ESR spectroscopy. Radiation Physics and Chemistry, 150, 35-39.

Werner, D. A., Straub, I., Zumsteeg, V., \& Kuntz, F. (2020). Identification of bleached and irradiated walnuts and hazelnuts by ESR spectroscopy. Radiation Physics and Chemistry, 173108882.

WHO (1999). High dose irradiation: Wholesomeness of food irradiated with doses above 10 kGyGeneva, Switzerland: WHO WHO Technical Report Series 890.

Yordanov, N. D., Lagunov, O., \& Dimov, K. (2009). EPR spectra induced by gamma-irradiation of some dry medical herbs. Radiation Physics and Chemistry, 78, 277-280.

Linke, B, Ammon, J, Ballin, U, Brockmann, R, Brunner, J, Delincée, H, Eisen, S, Erning, D, Eschelbach, H, Estendorfer-Rinner, S, Fienitz, B, Frohmuth, G, Helle, N, Holstein, A, Jonas, K, Krolls, W, Kunn, T, Kruspe, W, Marchioni, E, Meier, W, Pfordt, J, Schleich, C, Stewart, E, Trapp, C, Vreden, N, Wiezorek, C, Bogl, K W, Schreiber, G A, 1996. Elektronen-spinresonanzspektroskopische Untersuchungen zur Identifizierung bestrahlter getrockneter und frischer Fruchte: Durchfuhrung eines Ringversuchs an getrockneter Feigen und Mangos sowie an frischen Erdbeeren. Report of the Federal Institute for Health Protection of Consumer and Veterinary Medicine. BfVV-Heft 03/ 1996 (Bundesinstitut fur gesundheitlichen Verbaucherschutz und Veterinarmedizin, Berlin.

Raffi, J, 1992. Electron Spin Resonance Intercomparison Studies on Irradiated Foodstuffs. BCR-Information. Luxembourg: Commission of the European Communities, Report EUR/13630/en. 